\title{
Andreev Spectroscopy in Three-Terminal Hybrid Nanostructure
}

\author{
G. MichaŁeK $^{a, *}$, B.R. BuŁka ${ }^{a}$, M. URbaniak ${ }^{a}$, T. Domański ${ }^{b}$ And K.I. Wysokiński ${ }^{b}$ \\ ${ }^{a}$ Institute of Molecular Physics, Polish Academy of Sciences, M. Smoluchowskiego 17, 60-179 Poznań, Poland \\ ${ }^{b}$ Institute of Physics, M. Curie-Skłodowska University, pl. M. Curie-Skłodowskiej 1, 20-031 Lublin, Poland
}

We consider a hybrid three-terminal structure consisting of a quantum dot coupled to three leads, two normal and one superconducting. The current flowing between one of the normal and the superconducting electrodes induces voltage in the other normal (floating) electrode. The value of the induced voltage depends on the position of the Andreev levels in the quantum dot and is a measure of the interplay between the electron transfer and the subgap anomalous reflection processes. When the crossed (i.e. non-local) Andreev reflections dominate the induced potential in the normal electrode becomes negative. This situation occurs for the relatively strong coupling to the superconducting electrode and only outside the Coulomb blockade region. Taking the superconducting electrode as the voltage probe one gets additional information on competition of injected carriers.

DOI: 10.12693 /APhysPolA.127.293

PACS: $73.63 . \mathrm{Kv}, 73.23 . \mathrm{Hk}, 74.45 .+\mathrm{c}$

\section{Introduction}

The study of hybrid nanostructures is important because of their potential application in nanoelectronic, spintronic and quantum computation processing devices [1]. Here we consider a quantum dot attached to three external terminals, one of which is superconducting $(\mathrm{S})$ and two other normal $(\mathrm{N})$. Due to the presence of superconducting electrodes the pairing potential is induced in a quantum dot and the Andreev reflection (AR) processes contribute to the transport of electrons (e) and holes (h). In such hybrid structure direct (DAR) and crossed (CAR) Andreev processes appear to compete with normal electron transfers (ET). For ET the electron from one of the normal leads (say left one $-\mathrm{L}$ ) tunnels to the quantum dot and later directly to the right $(\mathrm{R})$ electrode. The CAR processes also involve $\mathrm{L}$ and $\mathrm{R}$ leads, however, e.g. the electrons from both leads form a Cooper pair and end up in the superconducting electrode.

The study of the voltage induced in one of the electrodes (called floating) due to the voltage bias applied between the remaining two electrodes is a source of information about the Andreev states induced in the dot and thus may be further used to get the information about the interactions and couplings between the dot and the leads. In the floating electrode the voltage builds up to prevent the flow of the current.

In the previous paper we have studied the same system and derived [2] the formulae for the currents flowing for the voltages in the subgap regime $|e V| \ll \Delta$. Under this condition the total current $J_{\lambda}$ in the lead $\lambda$ reads

$$
J_{\lambda}=J_{\lambda}^{\mathrm{ET}}+J_{\lambda}^{\mathrm{DAR}}+J_{\lambda}^{\mathrm{CAR}} \text {. }
$$

\footnotetext{
${ }^{*}$ corresponding author; e-mail: grzechal@ifmpan.poznan.pl
}

We have calculated the electrical currents $J_{\lambda}$ in the $\mathrm{N}$ lead $\lambda=\{L, R\}$ by use of the nonequilibrium Green function technique [3] and got

$$
\begin{aligned}
& J_{\lambda}^{\mathrm{ET}}=\frac{2 e}{h} \Gamma_{\lambda} \Gamma_{\alpha} \int_{-\infty}^{\infty} \mathrm{d} E\left|G_{11}^{\mathrm{r}}\right|^{2}\left(f_{\lambda}^{\mathrm{e}}-f_{\alpha}^{\mathrm{e}}\right), \\
& J_{\lambda}^{\mathrm{DAR}(\mathrm{CAR})}=\frac{2 e}{h} \Gamma_{\lambda} \Gamma_{\lambda(\alpha)} \int_{-\infty}^{\infty} \mathrm{d} E\left|G_{12}^{\mathrm{r}}\right|^{2}\left[f_{\lambda}^{\mathrm{e}}-f_{\lambda(\alpha)}^{\mathrm{h}}\right] .
\end{aligned}
$$

$\Gamma_{\lambda(\alpha)}$ denotes tunneling rate from the normal lead $\lambda$ $(\alpha), f_{\lambda}^{\mathrm{e}} \equiv f_{\lambda}^{\mathrm{e}}(E)=\left\{\exp \left(\left(E-\mu_{\lambda}\right) / k_{\mathrm{B}} T_{\lambda}\right)+1\right\}^{-1}$ is the Fermi-Dirac distribution function for electrons (e) in the lead $\lambda$ with the chemical potential $\mu_{\lambda}=E_{\mathrm{F}}+e V_{\mathrm{S}}+e V_{\lambda}$ and $f_{\lambda}^{\mathrm{h}} \equiv f_{\lambda}^{\mathrm{h}}(E)=1-f_{\lambda}^{\mathrm{e}}(-E)$ is the distribution function for holes (h).

The matrix elements (in the Nambu space) of the Green function of the quantum dot coupled to the electrodes is calculated from appropriate Dyson equations and take forms

$$
\begin{aligned}
& G_{11}^{\mathrm{r}}=\frac{1 / g_{22}^{\mathrm{r}}+\mathrm{i} \Gamma_{\mathrm{N}} / 2}{\left(1 / g_{11}^{\mathrm{r}}+i \Gamma_{\mathrm{N}} / 2\right)\left(1 / g_{22}^{\mathrm{r}}+i \Gamma_{\mathrm{N}} / 2\right)-\Gamma_{\mathrm{S}}^{2} / 4}, \\
& G_{12}^{\mathrm{r}}=\frac{\Gamma_{\mathrm{S}} / 2}{1 / g_{22}^{\mathrm{r}}+\mathrm{i} \Gamma_{\mathrm{N}} / 2} G_{11}^{\mathrm{r}},
\end{aligned}
$$

where $\Gamma_{\mathrm{N}}=\Gamma_{\mathrm{L}}+\Gamma_{\mathrm{R}}, \Gamma_{\mathrm{S}}$ describes coupling to the $\mathrm{S}$ lead and $g_{i i}^{\mathrm{r}}$ are diagonal matrix elements of the isolated quantum dot. The Green function for an isolated, single-level $\left(\epsilon_{0}\right)$ QD in the presence of Coulomb interactions [3] $(U)$ is calculated within Hubbard I approximation [4] and reads

$$
g_{11(22)}^{\mathrm{r}}=\frac{1-n / 2}{E \mp \epsilon_{0}+\mathrm{i} 0^{+}}+\frac{n / 2}{E \mp \epsilon_{0} \mp U+\mathrm{i} 0^{+}},
$$

where $n$ is the total charge accumulated at QD. $n$ depends on the full Green function and is calculated in the self-consistent way [2].

\section{Results}

\subsection{Floating normal lead}

First we will study the system, in which the $\mathrm{R}$ lead is floating and the bias is asymmetric, i.e. voltage $V_{\mathrm{L}}$ 
is applied only to the $\mathrm{L}$ lead, while $\mathrm{S}$ lead is grounded (i.e. $V_{\mathrm{S}}=0$ ). It means that the total current from the $\mathrm{R}$ lead vanishes, i.e. $J_{\mathrm{R}}=J_{\mathrm{R}}^{\mathrm{ET}}+J_{\mathrm{R}}^{\mathrm{DAR}}+J_{\mathrm{R}}^{\mathrm{CAR}} \equiv 0$. For small voltages $V_{\lambda} \rightarrow 0$ (i.e. in the linear regime) and at $T=0$ one finds the voltage $V_{\mathrm{R}}$ induced at the floating $\mathrm{R}$ lead as

$$
\frac{V_{\mathrm{R}}}{V_{\mathrm{L}}}=\frac{\mathcal{G}_{0}^{\mathrm{ET}}-\mathcal{G}_{0}^{\mathrm{CAR}}}{\mathcal{G}_{0}^{\mathrm{ET}}+2 \mathcal{G}_{0}^{\mathrm{DAR}}+\mathcal{G}_{0}^{\mathrm{CAR}}},
$$

where $\mathcal{G}_{0}^{\kappa}=\mathrm{d} J_{\mathrm{R}}^{\kappa} / \mathrm{d} V_{\mathrm{R}}$ denotes conductance at $E_{\mathrm{F}}=0$ due to process $\kappa$. Using Green functions [Eqs. (4),(5)] the last equation can be rewritten as

$$
\frac{V_{\mathrm{R}}}{V_{\mathrm{L}}}=\frac{\Gamma_{\mathrm{L}}\left[4 \epsilon_{0}^{2} \epsilon_{U}^{2}+\left(\Gamma_{\mathrm{N}}^{2}-\Gamma_{\mathrm{S}}^{2}\right) \epsilon_{\mathrm{N}}^{2}\right]}{4 \Gamma_{\mathrm{L}} \epsilon_{0}^{2} \epsilon_{U}^{2}+\left[\Gamma_{\mathrm{L}} \Gamma_{\mathrm{N}}^{2}+\left(\Gamma_{\mathrm{L}}+2 \Gamma_{\mathrm{R}}\right) \Gamma_{\mathrm{S}}^{2}\right] \epsilon_{\mathrm{N}}^{2}},
$$

where $\epsilon_{U}=\epsilon_{0}+U, \epsilon_{\mathrm{N}}=\epsilon_{0}+\left(1-n_{0} / 2\right) U$ and $n_{0}=$ $n\left(E_{\mathrm{F}}=0\right)$.

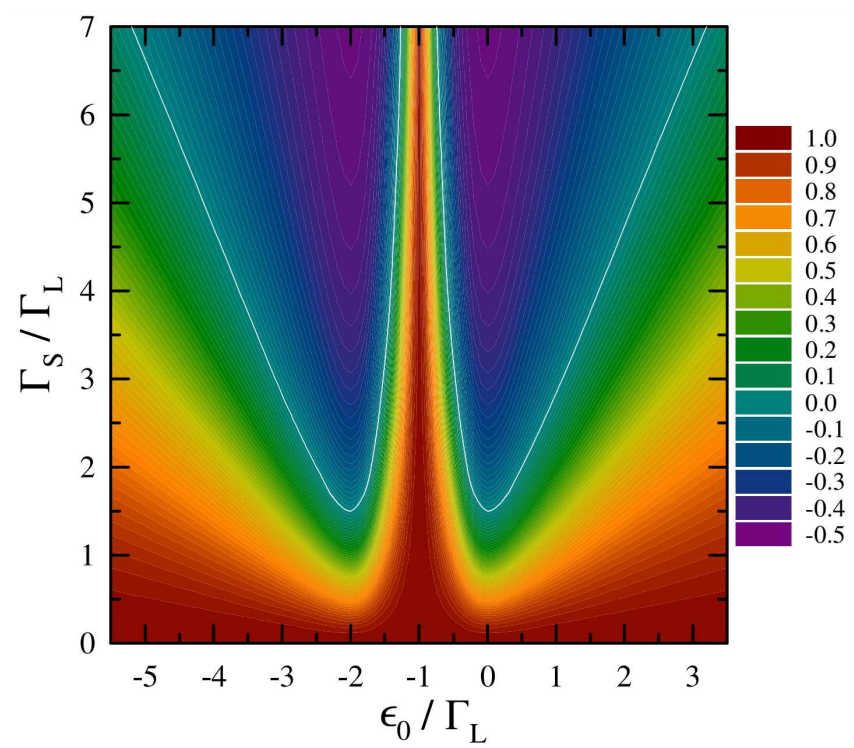

Fig. 1. Voltage $V_{\mathrm{R}} / V_{\mathrm{L}}$ induced (in the linear response regime) in the floating $\mathrm{R}$ lead plotted in the plane $\epsilon_{0}$ and $\Gamma_{\mathrm{S}}$ for the Coulomb interaction $U / \Gamma_{\mathrm{L}}=2$ and $\Gamma_{\mathrm{R}}=$ $0.5 \Gamma_{\mathrm{L}}$. The white curve shows $V_{\mathrm{R}}=0$, i.e. the border line at which $\mathcal{G}_{0}^{\mathrm{ET}}=\mathcal{G}_{0}^{\mathrm{CAR}}$.

Results of the numerical calculations of the $V_{\mathrm{R}} / V_{\mathrm{L}}$ in the linear regime are shown in Fig. 1 as a function of the $\epsilon_{0}$ and the coupling $\Gamma_{\mathrm{S}}$. One can see that the voltage $V_{\mathrm{R}}$ is sensitive to the pairing correlations induced in the QD by the proximity effect to the superconducting lead.

As long as $\Gamma_{\mathrm{S}}<\Gamma_{\mathrm{N}}$ the normal electron transfer (ET) processes dominate over non-local Andreev (CAR) scattering in whole voltage range $V_{\mathrm{L}}$, and the induced voltage $V_{\mathrm{R}}$ is of the same sign as $V_{\mathrm{L}}$. The situation is changed for the larger couplings $\Gamma_{\mathrm{S}}>\Gamma_{\mathrm{N}}$. In that regime CAR processes dominate over the ET processes $\left[\mathcal{G}_{0}^{\mathrm{CAR}}>\mathcal{G}_{0}^{\mathrm{ET}}\right.$, see Eq. (7)]. It is seen that when the e-h symmetry is broken (i.e. outside the Coulomb blockade region) the voltage $V_{\mathrm{R}}$ has opposite sign to the bias voltage $V_{\mathrm{L}}$. However, close to the particle-hole symmetry point, i.e. inside the CB region, the induced voltage $V_{\mathrm{R}} / V_{\mathrm{L}}$ is always positive and reaches its maximum value $V_{\mathrm{R}} / V_{\mathrm{L}}=1$. With an increase of the coupling $\Gamma_{\mathrm{S}}$ (increase of the on-dot pairing), the width of the $\mathrm{CB}$ region shrinks and so does the region of positive voltages $V_{\mathrm{R}} / V_{\mathrm{L}}$.

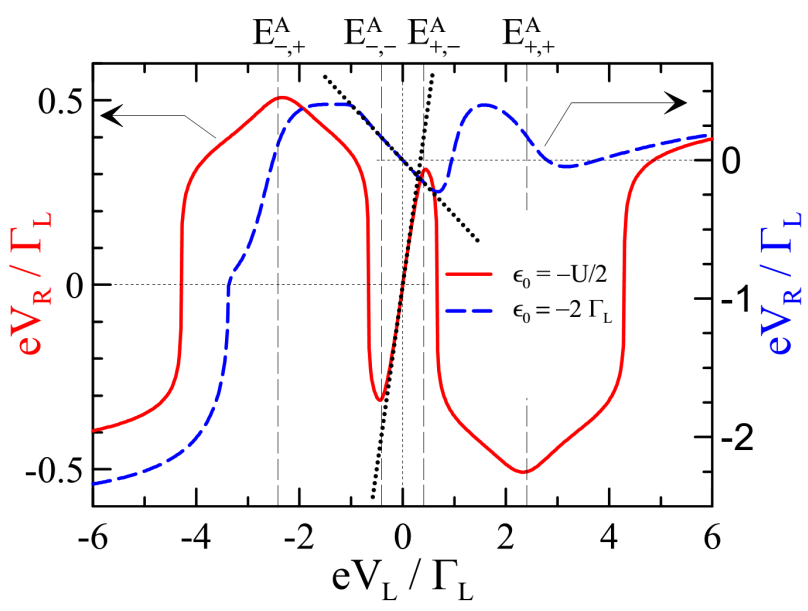

Fig. 2. Potential $e V_{\mathrm{R}}$ induced in the floating $\mathrm{R}$ lead as a response to applied bias voltage $e V_{\mathrm{L}}$ for $\epsilon_{0}=-U / 2$ (red solid line) and $\epsilon_{0}=-2 \Gamma_{\mathrm{L}}$ (blue dashed line). The other parameters are: $\Gamma_{\mathrm{R}}=\Gamma_{\mathrm{L}} / 2, \Gamma_{\mathrm{S}}=4 \Gamma_{\mathrm{L}}$, $U=2 \Gamma_{\mathrm{L}}$, and $V_{\mathrm{S}}=0$. The vertical lines (black, dashed) denote positions of the Andreev bound states $E_{\lambda, \lambda^{\prime}}^{A}=$ $\lambda \sqrt{\left(C+\lambda^{\prime} \delta\right) / 2}$, where $\delta=\sqrt{C^{2}-\left(\Gamma_{\mathrm{S}}^{2} \epsilon_{\mathrm{N}}^{2}+4 \epsilon_{0}^{2} \epsilon_{U}^{2}\right)}$, $C=\epsilon_{0}^{2}+\epsilon_{U}^{2}+\Gamma_{\mathrm{S}}^{2} / 4$ and $\lambda, \lambda^{\prime}= \pm 1$ for $\epsilon_{0}=-U / 2$. The dotted (black) line show $e V_{\mathrm{R}} / \Gamma_{\mathrm{L}}$ in the linear regime [calculated from Eq. (8)].

In the non-linear case the dependence $V_{\mathrm{R}}\left(V_{\mathrm{L}}\right)$ is more complicated. In Fig. 2 we have plotted the voltage induced in the floating $\mathrm{R}$ lead as a function of the bias voltage $V_{\mathrm{L}}$ applied to the system for the $\mathrm{e}-\mathrm{h}$ symmetric case $\epsilon_{0}=-U / 2$ and for $\epsilon_{0}=-2 \Gamma_{\mathrm{L}}$. In the case $\epsilon_{0}=-U / 2$ the voltage $V_{\mathrm{R}}$ is an antisymmetric function of $V_{\mathrm{L}}$. For small biases $\left|V_{\mathrm{L}}\right|$ the induced potentials $V_{\mathrm{R}}$ are linear functions of $V_{\mathrm{L}}$ (see black dotted lines close to $V_{\mathrm{L}}=0$ ) in agreement with the values obtained from Eq. (8). In this region none of the four Andreev bound states (vertical dashed lines in Fig. 2) is close enough to the chemical potential of the electrodes, so the currents are suppressed due to the Coulomb blockade. We have also found that when the Coulomb interactions $U$ increase, the amplitude of the induced voltage $V_{\mathrm{R}}$ is larger and the Coulomb blockade region becomes wider (not shown). Similarly, to the situation in the linear regime, the voltage $V_{\mathrm{R}}$ has always the same sign as the bias voltage $V_{\mathrm{L}}$. Near the Coulomb blockade border the nonlinearity becomes important. Let us analyze $V_{\mathrm{L}}<0$. In this region the current $J_{\mathrm{R}}^{\mathrm{AR}}=J_{\mathrm{R}}^{\mathrm{DAR}}+J_{\mathrm{R}}^{\mathrm{CAR}}<0$ compensates the current $J_{\mathrm{R}}^{\mathrm{ET}}>0$ for the negative voltage $V_{\mathrm{R}}$. When the voltage $V_{\mathrm{L}}$ crosses the Andreev bound state $E_{-}^{\mathrm{A}}$ - the induced voltage $V_{\mathrm{R}}$ has a local minimum. Further decrease of the $V_{\mathrm{L}}$ below $E_{-,-}^{\mathrm{A}}\left(e V_{\mathrm{L}}<E_{-,-}^{\mathrm{A}}\right)$ leads to an increase of the current $J_{\mathrm{R}}^{\mathrm{DAR}}>0$ and to a change of sign of the induced $V_{\mathrm{R}}$ from negative to positive. The current $J_{\mathrm{R}}^{\mathrm{DAR}}$ as well as the voltage $V_{\mathrm{R}}$ increase as long as $e V_{\mathrm{L}}>E_{-,+}^{\mathrm{A}}$. 
For $e V_{\mathrm{L}}=E_{-,+}^{\mathrm{A}}$ the maximal amplitude of the induced voltage $e V_{\mathrm{R}}$ can even exceed that for $e V_{\mathrm{L}}=E_{+,-}^{\mathrm{A}}$. When the $e V_{\mathrm{L}}$ decreases further, the Andreev bound state $E_{-,+}^{\mathrm{A}}$ becomes available for ET processes and the induced voltage $V_{\mathrm{R}}$ decreases. In the limit of the very large negative bias voltage $e V_{\mathrm{L}} \ll E_{-,+}^{\mathrm{A}}$ the currents $J_{\mathrm{R}}^{\mathrm{ET}}$ and $J_{\mathrm{R}}^{\mathrm{CAR}}$ saturate.

When e-h symmetry is broken (e.g. for $\epsilon_{0}=2 \Gamma_{\mathrm{L}}$ ) the induced potential $V_{\mathrm{R}}$ is asymmetrical function of the applied bias $V_{\mathrm{L}}$. The generated voltage $V_{\mathrm{R}}$ is smaller than in the $\mathrm{e}-\mathrm{h}$ symmetric case except the large negative voltage $V_{\mathrm{L}}$, when its amplitude is enhanced, see Fig. 2.

\subsection{Floating superconducting lead}

We consider now the floating S-lead. The total current $J_{\mathrm{S}}$ is given by Kirchhoff's law, so the potential $e V_{\mathrm{S}}$ induced in the $\mathrm{S}$ lead can be found from vanishing of the current $J_{\mathrm{S}} \equiv J_{\mathrm{L}}^{\mathrm{DAR}}+J_{\mathrm{R}}^{\mathrm{DAR}}+J_{\mathrm{L}}^{\mathrm{CAR}}+J_{\mathrm{R}}^{\mathrm{CAR}}=$ 0 . This may be rewritten in the form $J_{\mathrm{S}}=\Gamma_{N}$ $\int \mathrm{d} E\left[\Gamma_{\mathrm{L}} F_{\mathrm{L}}(E)+\Gamma_{\mathrm{R}} F_{\mathrm{R}}(E)\right]\left|G_{12}^{\mathrm{r}}(E)\right|^{2}$, where $F_{\lambda}(E)=$ $\sinh \left(x_{\lambda} / k_{\mathrm{B}} T_{\lambda}\right) /\left[\cosh \left(E / k_{\mathrm{B}} T_{\lambda}\right)+\cosh \left(x_{\lambda} / k_{\mathrm{B}} T_{\lambda}\right)\right]$ and $x_{\lambda}=e V_{\mathrm{S}} \pm e V / 2$ for $\lambda=L, R$. For the particle-hole symmetric situation the integrand is an even function of $E$ and odd function of $x_{\lambda}$. As a result the currents nearly vanish for voltages $|e V|<E_{+,-}^{\mathrm{A}}$ (region of the Coulomb blockade) and the potential induced in the floating $\mathrm{S}$ lead is strongly suppressed. In the linear limit (small $V_{\mathrm{S}}$ and $V$ and $\left.x_{\lambda}\right)$ one finds $V_{\mathrm{S}} / V=(1 / 2)\left(\Gamma_{\mathrm{R}}-\Gamma_{\mathrm{L}}\right) /\left(\Gamma_{\mathrm{L}}+\Gamma_{\mathrm{R}}\right)$. Figure 3 shows the induced potential $e V_{\mathrm{S}}$ as a function of bias voltage $\mathrm{eV}$ across the normal branch of the system outside the linear limit. The overall roughly linear $V_{\mathrm{S}}(V)$ dependence is caused by the broken $\mathrm{e}-\mathrm{h}$ symmetry $\left(\epsilon_{0} \neq-U / 2\right)$ together with an asymmetrical coupling $\Gamma_{\mathrm{R}} \neq \Gamma_{\mathrm{L}}$.

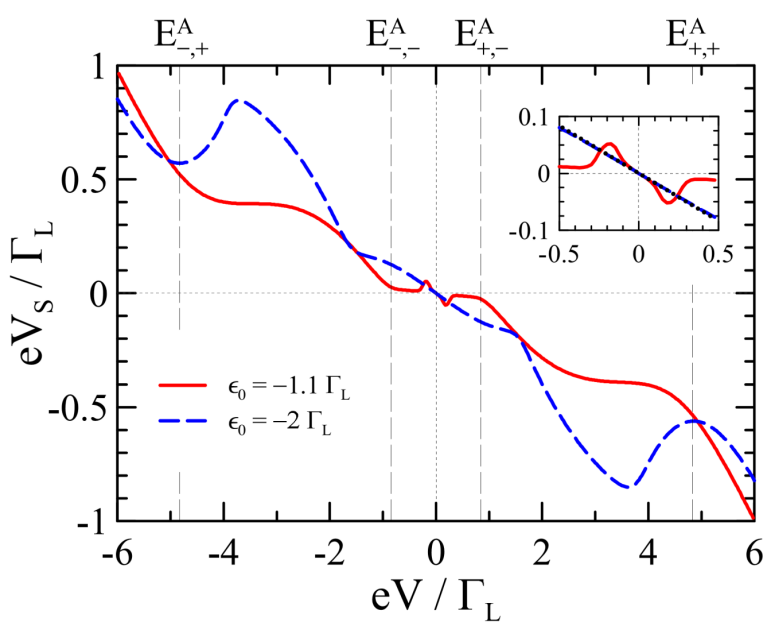

Fig. 3. Potential $e V_{\mathrm{S}}$ induced in the floating electrode vs. applied bias voltage $e V$ for $\epsilon_{0}=-1.1 \Gamma_{\mathrm{L}}$ (red solid line) and $\epsilon_{0}=-2 \Gamma_{\mathrm{L}}$ (blue dashed line). The other parameters are: $\Gamma_{\mathrm{R}}=\Gamma_{\mathrm{L}} / 2, \Gamma_{\mathrm{S}}=4 \Gamma_{\mathrm{L}}, U=2 \Gamma_{\mathrm{L}}, V_{\mathrm{L}}=$ $V / 2$ and $V_{\mathrm{R}}=-V / 2$. The region around $\mathrm{eV}=0$ is shown in the inset. The vertical lines (black, dashed) denote position of the Andreev bound states for $\epsilon_{0}=$ $-1.1 \Gamma_{\mathrm{L}}$.
In order to gain additional insight into the behavior of the induced voltage $V_{\mathrm{S}}$ we have also analyzed different tunneling processes, which contribute to the current $J_{\mathrm{S}}$ (not shown). We have found that the linear dependence is due to a competition of DAR processes from the L lead with DAR processes from the $\mathrm{R}$ lead and CAR scattering. When the voltage $V$ decreases below the Andreev bound state $E_{-,-}^{\mathrm{A}}$ the induced potential $V_{\mathrm{S}}$ strongly increases. For very large bias voltages $e V \ll E_{-,+}^{\mathrm{A}}$, all Andreev bound states lie between $e V_{\mathrm{L}}$ and $e V_{\mathrm{R}}$. We have found that the tunneling processes from the $\mathrm{R}$ lead give constant contribution to the current $J_{\mathrm{S}}$. In this voltage region $\mathrm{CAR}$ is activated due to an enhancement of the processes in the electron channel while the tunneling of the holes is reduced. A decrease of the current $J_{\mathrm{L}}^{\mathrm{DAR}}$ is a combined effect of an activation of the electron tunneling processes and a suppression of the tunneling of holes. On the other hand when $\mathrm{eV} \gg E_{+,+}^{\mathrm{A}}$ the roles of CAR and DAR processes are reversed.

In contrast to the case with the floating $\mathrm{N}$ lead, where the sign of the induced voltage $V_{\mathrm{R}} / V_{\mathrm{L}}$ for the given $\Gamma_{\mathrm{R}} / \Gamma_{\mathrm{L}}$ could be reversed by changing $\Gamma_{\mathrm{S}}$, the sign of the induced voltage $V_{\mathrm{S}}$ in the superconducting electrode depends on the difference $\left(\Gamma_{\mathrm{R}}-\Gamma_{\mathrm{L}}\right)$ in the linear regime.

In our studies of floating electrodes the discussion have been restricted to zero temperature limit. The voltage induced in the (floating) normal electrode changes sign with respect to the applied one if the non-local crossed Andreev reflections are dominant. This may be used as a sensitive probe of the CAR processes. More general analysis of the transport properties of three-terminal device will be published elsewhere [5].

\section{Acknowledgments}

The work has been partially supported by NCN under the contracts DEC-2011/01/B/ST3/04428 and DEC2012/05/B/ST3/03208.

\section{References}

[1] A. Martín-Rodero, A. Levy Yeyati, Adv. Phys. 60, 899 (2011).

[2] G. Michałek, B.R. Bułka, T. Domański, K.I. Wysokiński, Phys. Rev. B 88, 155425 (2013).

[3] H. Haug, A.-P. Jauho, Quantum Kinetics in Transport and Optics of Semiconductors, 2nd ed., Springer Verlag, Berlin 2008.

[4] J. Hubbard, Proc. R. Soc. Lond. A 281, 401 (1964).

[5] G. Michałek, B.R. Bułka, M. Urbaniak, T. Domański, K.I. Wysokiński, to be published. 